\title{
Über multiple Sklerose und herdförmige Enzephalitis.
}

\author{
Von \\ Prof. Friedrich Schnltze \\ in Bonn.
}

(Mit う Abbildungen.)

1.

Sklerotische Rückenmarksherde bei progressiver Paralyse.

Die kürzlich im Archiv für Psychiatrie und Nervenkrankheiten Bd. 60 erschienene Arbeit von Walter Riese über „Rückenmarksveränderungen eines Paralytikers" gibt mir Veranlassung, noch einmal auf eine im Jahre 1880 erschienene eigene Arbeit ${ }^{1}$ ) über den gleichen Gegenstand zurückzukommen. Diese allerdings fast 40 Jahre zurückliegende Mitteilung hat selbstverständlich ihre Lüicken, weil die mikroskopische Untersuchung nicht mit den heutigen Methoden ausgeführt werden konnte, gerade so gut, wie die heute veröffentlichten Arbeiten über den gleichen Gegenstand nach weiteren 40 Jahren ebenfalls ihre Lücken und Mängel aufweisen werden.

Aber im wesentlichen deckt sich mein damaliger, in zwei Fä̈llen ${ }^{2}$ ) erhobener Befund vollkommen mit dem neuesten Befund von Riese, und ich weiß nicht, warum von allen früheren Fällen nur der von ihm herbeigezogene von Brasch sich „mit Sicherheit auf eine echte progressive Paralyse" beziehen soll. Die klinische Diagnose war in dem einen meiner Fälle von vornherein, in dem andern erst im späteren Verłauf der mit den Erscheinungen von multipler Sklerose einsetzenden Erkrankung von einem 'so ausgezeichneten Psychiater wie Fürstner auf Dementia paralytica gestellt worden und wurde durch den von Arnold erhobenen makroskopischen und den von mir festgestellten mikroskopischen Gehirnbefund bestätigt.

1) Archiv f. Psych: Bd. 9, Heft 1.

2) Petroff erwähnt in seiner von Jolly beeinflußten Dissertation, ,Über die Beziehungen zwischen der multiplen Sklerose und Dementia paralytica " auffallenderweise nur den ersten dieser Fälle (Nik. Petroff, Iraug.-Diss. Berlin 1901). 
Aus den Abbildungen der Rückenmarksherde bei dem letzterwähnten Kranken, die sich in meinem Lehrbuche der Nervenkrankheiten S. 281 finden, geht klar hervor, daß es sich um echte sklerotische Herde, wie bei der multiplen Sklerose, gehandelt hat. Man bemerkt dasselbe scharfe Abschneiden der erkrankten Abschnitte gegen das übrige Gewebe wie stets bei dieser Erkrankung, dieselbe Unbekümmertheit um die graue und die weiße Substanz und um die einzelnen Stränge und Bahnen. Auch fehlte nach der von mir seinerzeit gegebenen Beschreibung trotz der sehr ausgedehnten, etwa $1 \mathrm{~cm}$ hohen Entartungszone innerhalb der ganzen Hinterstränge im Halsteil nebst angrenzenden Abschnitten eine deutliche Verfärbung der Gollschen Stränge oberhalb der erkrankten Abschnitte, also eine deutliche sekundäre Degeneration, wie sie an den wohlgelungenen, noch heute gut erhaltenen Weigertschen Präparaten hätte hervortreten müssen, wenn sie dagewesen wären.

In dem anderen Falle waren die Herde kleiner, aber ebenfalls von ganz unregelmäßiger Form und von ganz charakteristischer Beschaffenheit. Auch bei mittelstarken Vergxößerungen ist die erwähnte scharfe Abgrenzung der normalen von den erkrankten Abschnitten noch recht deutlich, und auch bei starken Vergrößerungen ist die Markscheidensubstanz in den längs getrofferien Nervenbahnen besonders innerhalb der grauen Substanz an vielen Stellen wie abgeschnitten, oder verdünnt sich wenigstens rasch bis zum Verschwinden, wie ich noch heute an den wohlerhaltenen Präparaten feststellen kann. Auf den Querschnitten durch die Hinter- und Seitenstränge ist oft eine schmale Grenzzone zwischen den kranken und gesunden Teilen zu finden, innerhalb deren die Nervenfasern nur zum Teile erhalten sind; innerhalb einer Pyramidenbahn des Dorsalteiles sind die Nervenfasern rarefidiziert.

Leider gestattete aber die damalige Technik noch nicht, in soichen Krankheitsherden markentblößte Achsenżylinder zu finden, wie mir das schon bald darauf im Jahre 1884 in einem im übrigen makroskopisch und mikroskopisch ganz gleichartigen Fallo. von multipler Sklerose ohne gleichzeitig paralytische Erscheinungen mit Hilfe der Fre ud schen Goldchloridmethode gelang. Ich habe ein Bild aus einem derartigen Herde in meinem erwähnten Buche (S. 282) der Darstellung des anatomischen Befundes bei multipler Sklerose beigegeben.

$\mathrm{K} . \mathrm{He} \mathrm{B}^{1}$ ) hat in einer unter meiner Leitung angestellten. Unter-

1) K. HeB, Arch. f. Psych. Bd. 19, S. 64. 
suchung ebenfalls mit dieser Methode gute Erfolge erzielt und Abbildungen seiner Befunde gegeben.

Es muß somit dahingestellt bleiben, wie viele Achsenzylinder sich in den Herden der von mir beschriebenen mit progressiver Paralyse verbundenen Fälle befanden, auf die übrigens auch in dem ganz neuen Falle von Riese nicht untersueht werden konnte. 'DaB sie zum Teile vernichtet wurden, geht mit Sicherheit daraus hervor, daß in der erkrankten grauen Substanz ein Fehlen von großen multiplen Ganglienzellen festgestellt wurde.

Auch in dem Falle von $\mathrm{He} B$ waren Ganglienzellen geschrumpft und hatten ihre ,feineren" Fortsätze verloren, wenn auch ,,vielleicht" bei einigen bereits stark atrophischen Ganglienzellen ein noch gut erhaltener Achsenzylinderfortsatz zu beobachten war. Da nun aber, wie aus der Zusammenstellung der anatomischen Befunde in der großen. Arbeit von Siemerling und Raecke ${ }^{1}$ ) im Archiv für Psychiatrie erhellt, eine Reihe von Untersuchern die Achsenzylinder bei multipler Sklerose in mannigfacher Art erkrankt und selbst vernichtet fanden, und nach den genannten beiden Autoren sogar die erste „mikroskopisch kleine Herdbildung mit umschriebenem Zerfall von Achsenzylindern" beginnen soll, dem sich erst ein umfassenderer Zerfall von Markscheiden anschlieBt, so ist dies Fehlen der Untersuchung auf Achsenzylinder in meinen beiden Fällen nicht von ausschlaggebender Bedeutung, zumal auch die Frage der Regeneration derselben nach längerem Bestehen der Erkrankung noch nicht endgültig entschieden ist. Es genügt, daß die nur geringfügige mikroskopisch nachweisbare sekundäre Degeneration auf das Fehlen einer größeren Anzahl von Achsenzylindern hinweist.

Eine größere Lücke in meiner erwähnten Arbeit besteht darin, daß das Großhirn und besonders seine Rinde nicht mit den heutigen Methoden untersucht werden konnte, und auch besonders nicht darauf untersucht wurde, was ja mit der Weige rtschen Markscheidenmethode ganz wohl möglich war, ob nicht jener eigentümliche ,Markfra B" der Rindenpartien vorhanden war, wie ihn Spielmeyer in seiner so interessanten Abhandlung ,Über einige anatomische Ähnlichkeiten zwischen progressiver Paralyse und multipler sklerose ${ }^{t i}$ beichreibt ${ }^{2}$ ).

Jedenfalls waren aber sowohl makroskopisch wie mikroskopisch

1) Siemerling u. Raecke, Bd. 53, S. 385 .

2) Zeitschr. f. d. ges. Neurol. u. Psych. Bd: 1, S. 660. 
in beiden Fällen die typischen Veränderungen der Dementia paralýtica. im Gehirn und in der Hirnrinde vorhanden, wie sie auch heutzutage noch als solche gelten. Makroskopisch sichtbare Herde von Sklerose fanden sich weder im frischen noch im gehärteten Gehirn.

Vor allem fällt aber für die Diagnose der progressiven Paralyse in meinem ersten Falle ins Gewicht, daß in der Vorgeschichte des Kranken eine sichere Syphilis vorhanden war. Der betreffende Kranke hatte 9 Jahre vor seiner Frkrankung, und zwar 1/4 Jahr nach seiner Verheiratung, seine Frau mit Syphilis angesteckt. Beide Ehegatten wurden gleichzeitig mit Schmierkuren behandelt. Bei dem zweiten Kranken konnte über das etwaige Vorhandensein einer syphilitischen Erkrankung nichts festgestellt werden.

Es ist somit, gerade so wie in dem Falle von Riese, durchaus wahrscheinlich, daß neben den sicheren durch die Lues hervorgerufenen Veränderungen in der Gehirnrinde auch die Rückenmarksherde im. Rückenmark durch die Einwirkung der Spirochacta pallida erzeugt worden sind. Es bleibt nur auffallend, daß ein derartiges Zasammenvorkommen so verhältnismäßig selten ist, zumal doch nach den Befunden von Spielmeyer in einem großen Prozentsatze der Fälle von Paralyse sich kleine multiple Herde von der grundsätzlich gleichen anatomischen Beschaffenheit wie derjenigen bei der multiplen Sklerose in der Hirnrinde, freilich seltener zugleich in der Marksubstanz des Gehirns vorfinden.

Vielleicht ist also doch in meinen und in ähnlichen Fällen ein zufälliges Zusammenvorkommen beider nicht so seltenen Erkrankunger vorhanden gewesen, also ein Gemisch beider, allerdings keine „Üben, gangsform", an die zu denken früher gewiß nicht unberechtigt warzumal ja auch bei der echten multiplen Sklerose die anscheinend gesunde Substanz der Sitz von diffusen Veränderungen sein kann, auch"in der Hirnrinde, wofür die so häufigen seelischen Veränderungen bei dieser Krankheit sprechen.

Mit Sicherheit wird sich die Frage erst beantworten lassen, wenn man, falls die Kuhn-Steinersche Spirochäte die Ursache der maltiplen Sklerose ist, diese neben der Pallida in den sklerotischen Herden oder überhaupt im zentralen Nervensystem vorfindet, oder vielleicht auch einen anderen Erreger der multiplen Sklerose, falls sich die Ku hnSteinersche Entdeckung nicht bestätigen sollte. Oder falls man eine gleich spezifische Reaktion für die multiple Sklerose im Blute, in der 
Lymphe oder in der Zerebrospinalflüssigkeit vorfindet, wie die Wasser mannsche Reaktion für die Lues.

Schließlich wäre eș aber auch möglich, daß der Krankheitserreger der multiplen Sklerose neben den gewöhnlich von ihm erzeugten multiplen Herden ähnliche Krankheitsprozesse diffuser Art im Gehirn und besonders in der Rinde hervorrufen könnte, wie die Spirochaeta pällida bei der progressiven Paralyse. Alles das kann erst die Zukunft lehren.

2.

Zur Lehre von den Ursachen der multiplen Sklerose.

In der neurologischen Bibel von Oppenheim (Lehrbuch der Nervenkrankheiten) wird es auch noch in der neuesten Auflage (S. 432) für ,zweifellos" erklärt, daß viele akute Infektionskrankheiten, „namentlich Typhus, Variola, Morbilli usw.", das Leiden im Gefolge haben können. Besonders habe man es nach Influenza sich entwickeln sehen. Auch an Cholera, Keuchhusten und akutem Gelenkrheumatismus schlösse sich eine echte multiple Sklerose an. Für einen von Henschen beschriebenen Fall, der sich nach Diphtherie entwickelte wird allerdings eher eine disseminierte Myelitis angenommen.

Aus dem Zusammenhange geht aber hervor, daß Oppenheim nicht bloß ein post hoc, sondern auch ein propter hoc annimmt, da ex weiterhin sagt, daß auch die Vergiftung mit metallischen Giften ,den Grund zu der Erkrankung" legen kann. Er mußte auch einmal den Einfluß eines jähen Temperatursturzes als „Ursache" beschuldigen.

Gegen diese seine und P. Marie's Annahme, daß so viele verschiedenartige Infektionskrankheiten die Ursache oder, um diesen Ausdruck zu vermeiden, die wesentliche Hauptbedingung für das Entstehen der echten, fortschreitenden multiplen Sklerose abgäben, sprechen folgende Gründe:

1. haben bekanntlich zahlreiche Untersucher eine derartige Beziehung zwischen bekannten Infektionskrankheiten und multipler Sklerose vermißt. Ich nenne besonders Strä̈mpell und J. Hoffman $n$. Marburg hält es in seiner Bearbeitung des Kapitels der multiplen Sklerose im Lewandowskyschen Handbuch der Neurologie mit Recht für "bishęr nicht erwiesen", daß derartige Infektionen eine ätiologische Rolle bei der multiplen Sklerose spielen, sondern vermag nur als sicher anzunehmen, da $\mathrm{h}$ sie wie hereditäre Veranlagung, 
Traumen und Vergiftungen ein bestehendes Laiden verschlimmern können.

Ich selbst habe aus der Gesa mtheit meiner Fälle zweimal aus meiner Klinik in Dissertationen über diese Beziehungen berichten lassen, früher von Elter ${ }^{1}$ ) (1897) und vor kurzem von Martin Eschweiler ${ }^{2}$ ) (1919). Unter den von Elter besprochenen 38 Fällen fand sich in der Vorgeschichte der Sklerotiker nur einmal Influenza angegeben, bei den 47 Kranken Eschweilers zweimal Influenza, zweimal Pneumonie und einmal Scharlach. In weiteren von mir selbst zusammengestellten 77 Fällen aus meiner Privatpraxis wurde in vielen eine vorhergegangene Infektionskrankheit geleugnet, nur in wenigen eine Vorkränkheit erwähnt. Ein 17jähriger Fabrikantensohn soll allerdings 40 mal Diphtherie gehabt haben, also wohl oft von Anginen heimgesucht worden sein. Gewiß mag manchmal, worauf $\mathrm{Nolda}^{3}$ ) hinweist, das Überstehen von Infektionskrankheiten besonders in der Kinderzeit vergessen und nicht angegeben worden sein. Aber ein unmittelbarer oder rascher Anschluß an derartige Erkrankungen würde doch schwerlich so häufig haben ïbersehen werden können.

2. Bei. einer Anzahl der angeschuldigten infektiösen Vorkrankheiten bestehen gar keine Beziehungen zwischen ihren eigentlichen Erregern und dem zentralen Nervensystem. Besonders Masern, Scharlach, Keuchhusten machen so gut wie niemals auch nur eine Meningitis, abgesehen von sehr seltenen Fällen von Mischinfektion mit Streptokokken, Staphylokokken. Das Diphtheriegift erzeugt zwar bekanntlich gewisse Erkrankungszustände im Nervensystem, aber keine herdförmige Entzündungen. Wie anders müßte das Krankheitsbild aller dieser Infektionskrankheiten aussehen, wenn ihre Erreger auch nur entfernt ähnliche Beziehungen zu den Ganglienzellen und Nervenfasern hätten, wie etwa die Lyssa-, Tetanus- und Poliomyelitiserreger?

1) Joh. Elter, Zar Ätiologie der multiplen Sklerose und der chron. Myelitis (Inaug.-Diss. Bonn 1897).

2) Martin Eschweiler, Utber die Ursachen der multiplen Sklerose (Inaug.Diss. Bonn 1919).

3) Nolda, Ein Fall von multipler Sklerose im Kindesalter usw. Arch. f. Psych. Bd. 23, S. 565. Bei dem von Nold a aus meiner Klinik veröffentlichten Falle waren den Krankheitserscheinungen im Gebiete des Nervensystems keine Infektionskrankheiten vorangegangen. Mit den Anschauungen des Verf. habe ich mich niemals einverstanden erklären können, schon wegen der Schwierigkeiten der Diagnose der multiplen Sklerose im Kindesalter. 
Dabei ist natürlich angesichts der vorliegenden Tatsachen nicht entfernt zu leugnen, da h sich ausnahmsweise besonders bei den Pocken, aber auch bei Typhus und Pneumonie, deren Erreger ja so oft eine Meningitis erzeugen, auch multiple entzündliche Herde im zentralen Nervensystem entwickeln können. Nach dieser Richtung hin sind nach wie vor die schönen Beobachtungen von $\mathrm{C}$. Westphal über die ,fleckweise oder disseminierte Myelitis" nach Pocken ${ }^{1}$ ) von großem Wert. Aber bei diesen herdförmigen akuten Entzïndungen handelt es sich gewöhnlich um eine einmalige Erkrankung, die rasch einsetzt, rasch ihren Höhepunkt erreicht und dann stationär bleibt, falls sie nicht, wie in den Westphalschen Fällen, wegen ihrer Schwere und Ausbreitung bald zum Tode führen. Es fehlt das dauernde Fortschreiten der Krankheitserscheinungen, besonders auch dasjènige in Schüben mit oft jahrelangen Pausen wie bei der multiplen Sklerose.

Nun kann allerdings auch wohl einmal diese, auch wenn sie ohne jede sonstige bekannte vorangegangene Infektionskrankheit entstanden ist, nicht stetig oder in Schüben fortschreiten, sondern stationär bleiben oder gar ausheilen, wenn auch das noch nicht durch a natomische Untersuchungen erwiesen ist. Und andererseits ist es denkbar, daß sich aus unbekannten Gründen aus den scheinbar zur Ruhe gekommenen vernarbten Herden einer früheren akuten herdförmigen, nach den erwähnten akuten Infektionskrankheiten entstandenen Enzephalomyelitis neue Krankheitsherde entwịckeln, wie das in äußerst seltenen Fällen auch nach der akuten Poliomyelitis vorzukommen scheint. Es, könnten z. B. irgendwo Krankheitserreger zurückgeblieben sein, wobei ich natürlich von den chronischen Infektionskrankheiten, wie Tuberkulose und Syphilis, absehe.

Auch könnten bei dem Vorhandensein von Resten dieser früher vorhanden gewesenen disseminierten Enzephalomyelitiden die von ihnen herrührenden Folgeerscheinungen anscheinend den Eindruck einer fortschreitenden Erkrankung machen, wenn ohne jede weitere Ausbreitung der einmal entstandenen Herde selbst die alten Krankheitserscheinungen, wie Lähmungen, sịch verstärken oder sich Zystitis oder Dekubitus hinzugesellen, falls z. B. allgemeine Ernährungsstörungen, unzweckmäßige Lebensweise, anderweitige Erkrankungen u. dgl. einwirken.

1) C. Westphal, Beobachtungen und Untersuchungen über die Krankheiten des zentralen Nervensystems. Arch. f. Psych. Bd. 4, S. 338ff. 
Es kann somit manchmal schwierig oder sogar unmöglich sein, zwischen echter multipler Sklerose und einer disseminierten akuten herdförmigen Enzephalomyelitis, die in ein chronisches Stadium übergetreten ist, mit Sicherheit zu unterscheiden.

Aber diese Schwierigkeit ändert nichts an der Tatsache, daß in der ungeheuer großen Mehrzahl der Fälle diese Unterscheidung vollkommen sicher zu machen ist.

3. Daß eine Menge so verschiedener akuter Infektionskrankheiten, wie das angègeben wird, allesamt eine anatomisch wie klinisch so eigenartige Erkrankung erzeugen: soll, wie die multiple sklerose, ist recht schwierig anzunehmen. Darauf hat Ed. Müller in seiner bekannten Monographie über diese Krankheit mit Recht hingewiesen. Schon lange habe ich deswegen an die „Existenz besonderer Krankheitskeime oder Stoffe gedacht", die im zentralen Nervensystem oder irgendwo im Körper verbleiben können und sich gelegentlich weiter verbreiten. Ich habe diese Meinung in meinem Referate über chronisch-organische Hirn- und Rückenmarksaffektionen im Jahre 1910 zum Ausdrucke gebracht ${ }^{1}$ ). Von gleichen Voraussetzungen ausgehend, haben offenbar Kuhn und Steiner ihre bekannten Untersuchungen begonnen, deren Vollendung und endgültige Bestätigung noch aussteht.

Nehmen wir die Richtigkeit der Befunde dieser Forscher an, so würde in ana tomischer Beziehung zum Teil eine verblüffende Ähnlíchkeit mit der Wirkungsweise einer anderen Spirochäte, der Pallida, gegeben sein. Denn nach den Untersuchungen Spielmeyers finden sich ja gar nicht selten bei der Dementia paralytica viele kleine Herde mit marklosen Flecken und Erhaltenbleiben der Achsenzylinder; allerdings vorzugsweise in der Hirnrinde, aber auch auf die weiße Substanz über-greifend. Zum Unterschiede von den Frregern bei der multiplen Sklerose zerstören also die Luesspirochäten mehr die Rindensubstanz als die weiße und exzeugen vor allem mehr diffuse Veränderungen. Indessen kommen auch bei der multiplen Sklerose neben den eigentlichen Herden „diffuse sklerotische Veränderungen" vor, wie z. B. in ausgesprochener Weise in dem erwähnten Falle, den Karl Heß beschrieben hat und der von Erb und mir klinisch beobachtet war. Zeichen von Dementia paralytica waren bei diesem Kranken auch kurz vor seinem Tode nicht bemerkt worden.

Auch klinisch sind die Ähnlichkeiten zwischen beiden Erkran-

1) Diese Zeitschrift Bd. 38 . 
kungen oft große. Beide können z. B. akut, sogar mit a poplektischen Lähmungen oder epileptischen Anfällen beginnen; auch bei der multiplen Sklerose sind oft genug seelische Störungen, besonders auch in Form der Demenz und Euphorie, vorhanden, und vor allem gibt es bekanntlich auch bei der progressiven Paralyse nicht selten starke Remissionen und selbst anscheinende oder wirkliche Heilungen. Selbst eine Lichtstarre der Pupillen ist bei der maltiplen Sklerose von gùten Beobachtern festgestellt worden (Uhthoff, Marburg und Anderen).

Andererseits muß man Strümpell durchaus beipflichten, wenn er in seinen „Bemerkungen zur Ätiologie der multiplen Sklerose "1) hervorhebt, daß wix auch, die endgültige Bestätigung der Kuhn-Steinerschen Befunde vorausgesetzt, noch keine wirklich befriedigende Einsicht in die Pathogenese der Krankheit haben, wie wir ja auch von einem „, wirklich vollständigen Einblick in die Entstehung der Tabes noch ziemlich weit" entfernt sind. In de m Punkte kann ich allerdings Str ümpell nicht beistimmen, daß er meint, es spiele das Moment der Infektiosität, das der Ansteckung, bei der multiplen Sklerose gar keine Rolle. Nie beobachte man die Krankheit bei Geschwistern oder bei Ehegatten, nirgends finde sich ein Hinweis auf ein endemisches oder gar epidemisches Auftreten der Krankheit. Das letztere ist, soweit bis jetzt bekannt, richtig; ein Auftreten bei Geschwistern ist aber hier und da beobachtet worden, besonders von J. Hoffmann, der die Fälle von familiärem Aúftreten der Erkrankung zusammengestellt hat ${ }^{2}$ ).

4. Ein vierter Grund gegen die Auffassung, daß alle möglichen Infektionskrankheiten bekannter Art eine echte multiple Sklerose hervorrufen, ist endlich der, daß in den verhältnismäßig wenigen Fällen, in denen von den Kranken oder ihren Angehörigen angegeben wurde, da $\beta$ sich die auf multipler Sklerose bezogenen Krankheitserscheinungen unmittelbar oder kurze Zeit an die beschuldigte Grundkrankheit anschlossen, eine Untersuchung vor der Erkrankung auf alle jene Frühzeichen der multiplen Sklerose fehlt, die wir jetzt kennen. Es könnte also sich immer nur; wie auch Marburg hervorhebt, um eine Verschlimmerung der schon früher vorhandenen Erkrankung gehandelt haben. Am deutlichsten tritt das nach meiner Erfahrung gelegentlich dann in die Erscheinung, wenn während eines Wochenbettes die anscheinend ersten Krankheitserscheinungẹn der multiplen Sklerose auf-

1) Strümpell, Neurol. Zentralblatt 1918, Nr. 12.

2) J. Hoffmann, diese Zeitschr. Bd. 47 u. 48, S. 247. 
treten. In einem solchen, von mir beobachteten Falle stellte sich heraus, daß schon viel früher einmal eine retrobulbäre Neuritis beobachtet worden war.

Immerhin ist dieser letzte Gegengrund kein positiver und kein entscheidender.

Auch für die stets von neuem in der Liceratur erwähnte Auffassung Oppenheims, daßeine Vergiftung mitme tallis chen Giften ,, den Grund zur Entstehung der multiplen Sklerose legen könne", ließ sich in meinen Fällen keine Unterlage finden, ebensowenig wie in denjenigen so vieler anderer Untersucher. Dagegen ist 0 p penhei $\mathrm{m}$ durchaus beizustimmen, wenn er die nach Kohlenoxydvergiftung auftretenden, zuweilen sogar fortschreitenden Krankheitserscheinungen nicht als multiple Sklerose auffaßt. Das Krankheitsbild ist vièmehr nur ein ähnliches wie dasjenige der multiplen Sklerose, wie das schon Stursberg ${ }^{1}$ ) in einem aus meiner Klinik veröffentlichten Falle betont hat und wie ich das ebenfalls angenommen habe und annehme. Es ist nur sehr bemerkenswert und wichtig, daß, wie Stursberg mit Recht hervorhebt, durch die von ihm beobachteten Vergiftungsfälle ,einwandfrei“ nachgewiesen wird, daß eine einmalig einwirkende Schädigung durch ein Gift ein fortschreitendes schweres Nervenleiden hervorrufen kann. Eine völlig ausreichende Erklärung für dieses Verhalten, die vielleicht auch auf die Einwirkung infektiöser Gifte in Betracht kommen könnte, ist noch nicht möglich. Ich möchte vor allem daran denken, daß bei der Einwirkung des Kohlenoxydgases auf das Gehirn mit seinen Gefäßen einzelne Teile desselben sofort sehr stark, andere abẹr schwächer betroffen werden. Die Betriebs- und Stoffwechselstörungen in diesen schwächer geschädigten Abschnitten können noch eine Zeitlang überwunden werden, später a ber nicht mehr. Wenn ein einmaliger starker Frost die Blätter eines Baumes trifft, so wird oft zunächst nur ein Teil der Blätter sofort vernichtet, ein anderer Teil aber noch eine Zeitlang funktionieren, schließlich aber auch erkranken und absterben. Dabei mögen noch Störungen durch die Funktion selbst, an die Stursberg denkt, oder auch irgendwelche schädigende sonstige Einflüsse in Frage kommen, die durch die vom Gift zunächst nur schwach veränderten Zellen nicht mehr wie von gesunden Zellen mit Erfolg abgewehrt werden können.

Steine ${ }^{2}$ ) hat ganz neuerdings mit aller gebotenen Vorsicht darauf

1) Stursberg, diese Zeitschrift Bd. 34, S. $432 \mathrm{ff}$.

2) Steiner, Neurol. Zentralbl. 1918, Nr. 15, S. 535. 
aufmerksam gemacht, daß vielfach Angehörige von Berufsschichten erkranken, die auf dem freien Lande arbeiten. Er glaubt nach seinen Erhebungen, daß Zeckenstiche eine Rolle für die Übertragung der Krankheitskeime spielen könnten. Er weist aber auch darauf hin, daß das vielen Ärzten zugängliche Krankheitsmaterial vorzugsweise von Menschen „niederer" Stände herrührt.

Was meine eigenen Erfahrungen in diesem Punkte angeht, so habe ich die Erkrañkung auch in den nicht handarbeitenden und in der städtischen wohlhabenden Bevölkerung nicht allza selten gesehen. In meiner Privatpraxis, in der der Mittelstand bei weitem überwiegt, fanden sich unter 77 Fällen zunächst die Fra uen in erheblicher Überzahl, nämlich 47 gegen 30 Männer, während in der Klinik in den beiden angegebenen Statistiken auf 27 Frauen 58 Männer kamen. Im ganzen zähite ich also 74 . Frauen gegen 88 Männer. Es besteht also, wie auch in anderen Statistiken, kein wesentlicher Unterschied der Geschlechter.

Das spricht schon allein dagegen, daß irgendwelche mit Gifteinwirkungen verbundenen Gewerbe eine Rolle spielen.

Viele der Kranken meiner Privatklientel stammten aus kleinen Städten, aber auch nicht wenige aus Großstädten, wie Köln und Düsseldorf. $\mathrm{Ob}$ die letzteren, besonders die Frauen, stets in ihnen gelebt hatten, und besonders in ihrer Kindheit, wurde nicht festge stellt. Aus dem Lande stammten zwei Gutsbesitzersfrauen, von denen die eine, die stets auf dem Lande gewohnt hatte, nack eingehender Befragung leugnete, jemals von zeckenähnlichen Tieren gebissen worden zu sein.

Aus den "Stubenhockerberufen " waren an multipler Sklerose erkrankt: 3 Lehrer, von denen allerdings 2 auf dem Lande wohnten, die wohl nicht als eigentliche Stubenhocker zu bezeichnen sind; ferner 7 Kaufleute, von denen 5 in Großstädten ansässig waren und einer ein junger wohlhabender Bankier war. Sodann eine musiktreibende Dame aus Bonn. Die Theologie "war durch einen Vikar, der Juristenstand durch einen Stud. jur. vertreten. Auffallenderweise fand sich kein Offizier unter den Erkrankten, obwohl sich die Angehörigen dieses Standes viel im Freien aufhalten. Andererseits wird auch dieser Beruf gar nicht erwählt, wenn sich, wie nicht selten, schon vor dem Eintrittsalter in ihn Erscheinungen der multiplen Sklerose eingestellt hatten.

Es läßt sich also aus dieser Sta tistik nichts in bezug auf eine etwaige Übertragung der Krankheit durch Zecken oder Insekten entnehmen. Sie zeigt nur, daß die Angehörigen der wohlhabenden und nicht handarbeitenden Bevölkerungsklassen verhältnismäßig nicht in geringerem 
Ma Be befallen wurden als die anderen, in Übereinstimmung mit den Erfahrungen von Otto Marburg.

3.

Zur Prognose der multiplen sklerose.

Je mehr und je länger man eine für gewöhnlich fortschreitende schwere Erkrankung kennen lernt, die naturgemäß zuerst in ihren ausgeprägten Formen bekannt wird und vielfach zuerst für unbedingt tödlich gilt, desto mehr lernt man allmählich auch weniger schwere und weniger ausgeprägte Fälle der gleichen Erkrankung kennen und desto vorsichtiger wird man mit der Vorhersage. 'Das war so bei der „perniziösen Anämie ", die durchaus nicht immer, ,perniziös" ist. Das war so in weit höherem Grade bei der Lungenschwindsucht. Und das ist in der Nervenpathologie bei dier Tabes, bei der Dementia paralytica und sogar bei der tuberkulösen Meningitis der Fall gewesen. Selbst gewisse, durch regelwidrige Anlage einzelner Organe und Organsysteme entstehende Erkrankungen, die gewölmlich ehernen Ganges bis zum Ende fortzuschreiten pflegen, können sich hier und da zuriickbilden, wie das z. B. Erb bei der Muskeldystrophie sah.

Was die multiple Sklerose angeht, so führen sowohl Oppenheim als auch Marburg, O. Maas und Bramwell, den Oppenheim zitiert, Fälle an, bei de nen man von Heilung sprechen dürfe, jedenfalls aber von sehr lange andauernder Besserung. So berichtet 0 . Maa s ${ }^{1}$ ) von einem ungewöhnlich günstigen Vérlauf in einem mittelschweren Falle, bei dem nach erheblicher Besserung nach 13 Jahren fast keine nervösen Beschwerden mehr bestanden und nur noch unbedeutende Reste der Erkrankungserscheinungen zurückgeblieben waren.

Allerdings kann man ebensowenig wie bei der progressiven Paralyse mit mathematischer Sicherheit behaupten, dab nicht selbst bei einer Pause von mehreren Jahrzehnten doch noch später ein Rückfall kommen könnte. Indessen ist es andererseits selbstverständlich, da $B$ bei einem schwerer Erkrankten gewisse Reste der früher überstandenen Krankheit zurückbleiben müssen und weiterhin sehr wahrscheinlich, daß bei leichter Erkrankten eine im praktischen Sinne völlige oder nahezu völlige Heilung eintreten kann. Dena so gut erfahrungsgemäß das Teilsymptom der retrobulbären Neuritis sich so zurückbilden kann, daß keine Sym-

1) O. Maas, Berliner klin. Wochenschr. 1907, Nr, 7, S. 197. 
ptome von ibr mehr nachzuweisen sind, so gut kann das auch für lange Zeit und selbst danernd bei gleichartigen Herderkrankungen an anderen Stellen des Zentralnervensystems der Fall sein.

Zu einem gewissen Vertrauen in die Latenz oder das Abgeheiltsein des krankhaften Prozesses wird man auch weiterhin dann berechtigt sein, wenn anerkannte Schädlichkeiten, wie z. B. das .Wochenbett, bei anscheinend Geheilten keinen Rückfall oder gar Fortschritt des.Leidens hervorriefen.

So untersuchte ich eine junge. Dame von etwa 20 Jahren, eine Architektentochter aus Bonn, als sie ohne jede äußere Veranlassung ausreichender Art drei epileptische Anfälle erlitten hatte:. Ihr Vater ist kräftig und gesund, ihre Mutter „,nervös“; sie selbst war früher stets gesund. Sie will sich bei einem längeren Aufenthalte in England durch das Erlernen der englischen Sprache und durch Sport sehr angestrengt haben, hatte aber niemals Zeichen von Hysterie. Die Anfälle begannen mit Augenverdrehen and führten zu Bewußtlosigkeit, konischen und klonischen Zuckungen und Zungenbiß. Bei dem zweiten Anfalle außer Zungenbiß noch Enuresis. Die Anfälle dauerten nur zwei Minuten und waren mit Verziehen der linken Gesichtshälfte verbunden. In den Zwischenpausen Wohlbefinden. Objektiv bei dem kräftigen, blühenden Mädchen keine Störungen auffindbar, auch kein Fazialisphänomen, wie es sich hier und da bei Epileptischen findet. Mitte 1919 Klagen über Parästhesien im rechten Mitteifinger, die mit angestrengtem Klavierspielen in Verbindung ge bracht wurden. Im Januar 1910 stellte Herr Prof. Hummelsheim ein zentrales Skotom $\mathrm{R}$ fest und eine retrobulbäre Neuritis. Im Jahre 1912 Wohlbefinden, keine Neuritis und kein Skotom mehr nachweisbar. Kein Nystagmus, die Patellarreflexe zwar recht lebhaft, aber nur von normaler Stelle aus zu bekommen. Kein Babinski; die Bauchdeckenreflexe aus äußeren Gründen nicht untersuchbar. Die Diágnose konnte nicht wohl anders als auf eine multiple Sklerose gestellt werden. Trotzdem wurde unter schweren Bedenken die Heirat zugelassen. Aber auch im Puerperium vor etwa 5 Jahren keine neuen Krankheitserscheinungen; auch jetzt, nach mindestens 7 Jahren, volle Gesundheit, die ich allerdings nicht durch eigene Untersuchung feststellen konnte.

Dieser Fall schließt sich also einem von Marburg a. a. O. erwähnten ganz ähnlichen an, in dem ebenfalls eine Heirat bei beginnender multipler Sklerose innerhalb einer allerdings wesentlich kürzeren Zeit der Beobachtung nicht schadete.

Ein lehrreiches. Gegenstück bot der folgende Fall:

Bei einem etwa 19jährigen jungen Mädchen, der in Bonn geborenen Tochter eines hohen Richters, war im Jahre 1895 von Herrn Prof. Pe te rs (jetzt Direktor der Augenklinik in Rostock) eine ,retrobulbäre axiale Neuritis" der Sehnerven festgestellt worden. Die Sehschärfe war auf $2 \%$ herabgesetzt. Rot und grün wurden im Zentrum leicht verschleiert gesehen. Nach Darreichung von Salizylpräparaten und unter: leichter Schwitzkur. 
versehwanden die Sehstörungen und die skotome wieder. Mit dem Augenspiegel nichts Regelwidriges zu. sehen. Im übrigen war das Mädchen stets gesund gewesen. Nach ihrer Verheiratung mit einem in einer Mittelstadt wohnenden Augenarzt entstand im Wochenbett im Jahre 1898 am 12. Tage Fieber, das auf eine Lymphdrüsenschwellung nach Brustwarzenschwunden bezogen wurde. Nach dem Aufstehen Gefühl von Schwere in beiden Beinen, das immer mehr zunahm, so daß im April 1914 schon nach 5 Minuten langem Gehen starke Müdigkeit eintrat. Augen und Blase gut; keine Schmerzen. Bei der Untersuchung deutlichste spastische Parese mit Fußklonus besonders links. An den Armen keine Reflexsteigerung, kein Nystagmus. Januar 1903 fand ich fast völlige Lähmung der Beine; nur die Wadenmuskeln noch leidlich kräftig. Das Gefühl für Tast-, Schmerz- und Temperaturreize an den Beinen und Füßen normal, nicht aber das Lagegefühl. Deutlichster Babinski, Fußklonus; die Patellarreflexe nur mäßig gesteigert. Die Armmuskeln leicht ermüdbar, deutliches Intentionszittern, Schwierigkeit beim Einfädeln und Zuknöpfen, aber keine Steigerung der Arníreflexe.

Kein Nystagmus, aber wieder schlechteres Sehen, Skotome. Abblassung der Schläfenhälfte und der Papillen (Augenarzt Dr. Hillesheim). Sprache regelrecht.

Also in diesem Falle trotz anscheinender Heilung der offenbar den Beginn des Lidens darstellenden retrobulbären Neuritis später der Eintritt von schweren Krankheitserscheinungen im Wochenbett, das mit leichter umschriebener fieberhafter Lymphdrïsenanschwellung verlief.

Zugleich lehrt der Fall auf das deutlichste, wie leicht voribergehende Störungen in einem trüheren Alter nach Ausheilung von den Erkranktgewesenen und ihren Angehörigen vergessen oder nicht erwähnt werden können, und wie leicht irgendeine fieberhafte Erkrankung oder ein Puerperium als die wesentliche Ursache oder Vorbedingung des Leidens erscheinen kann.

In einem weiteren in meinem Lehrbuche erwähnten typischen Falle den ich früher als geheilt ansah, ist zwar die Heilung 5 Jahre lang geblieben, aber es erscheint mir nach erneuter Durchsicht der Krankengeschichte die Diagnose nicht ganz sicher.

Diese Schwierigkeit bleibt auch für manchen anderen Fall bestehen und könnte eventuell erst durch eine Autopsie beseitigt werden, die noch Reste der früheren Krankheitsherde auffinden lassen müßte, oder besonders in den Frühstadien und in den atypischen Fällen dadurch, da 6 man eine spezifische Reaktion nach Art der Wassermannschen entdeckt. In drei aus meiner Klinik veröffentlichten Fällen ${ }^{1}$ ) von K. Voigt

1) Utber anscheinende Heilung von multipler Sklerose. Inaug.-Diss. von K. Voigt, Bonn 1901 . 
ist nur bei dem zweiten dort berichteten die Diagnose ganz sicher. Es. war aber bei ihm nur ein Teil der Krankheitserscheinungen zurückgegangen. In dem dritten lag höchstwahrscheinlich eine Hydrozephalie nach schwerem Trauma vor. Diese Fälle können somit für die Frage der Ausheilung der multiplen Sklerose nicht verwertet werden. Auch durch die etwaige Verknüpfung mit hysterischen Symptomen, die ihrerseits dauernd verschwinden können, wird das Urteil über etwaige :Ausheilung erschwert.

4.

Multiple kleinherdige fortschreitende Enzephalitis mit sehr geringer Gefäßbeteiligung (akute multiple Sklerose?).

\section{(Mit 5 Figuren.)1)}

Krankengeschichte: Der 43jährige Kranke, Herr Reg.-Rat. V., hat nach Angabe seines Hausarztes jahrelang an ,choreaähnlichen Muskelzuckungen ${ }^{\text {" }}$ gelitten, die in den letzten Jahren an Stärke erheblich abgenommen haben. Nach der Angabe seines Bruders war er ein „,nervöses" Kind und bekam nach Masern ein Zucken des Kopfes, das bei gutem Allgemeinbefinden später erheblich geringer wurde, aber stets bestehen blieb. Er war wegen dieser Zuckungen seit früher Kindheit oft in ärztlicher Behandlung, so bei Eulenburg, Wernicke, Hirt, und wurde elektrisiert und massiert. Im übrigen sei er ,prachtvoll gesund" gewesen, Bei seiner Mutter hatte sich im Klimakterium im Anschluß an starke Gemütserregungen eine vorübérgehende Psychose entwickelt. Eine Schwester der Mutter litt viel an Hysterie.

Er selber schrieb 1908 an seine Schwester, daß er vor einiger Zeit wieder "die partiellén leichte n Lähmungserscheinungen im linken Arm" gehabt habe, die er schon einmal früher (1894) nach heftiger Gemütserregung infolge der erwähnten: 11/2. Jahre dauernden Psychose seiner Mutter bekommen hatte. Nach ,einigér Zeit" verschwanden diese Lähmungserscheinungen wieder. Auch eine schon öfters nach Arbeit und Gemütserregungen entstanden sein sollende linksseitige Gesichtsneuralgie stellte sich wieder ein. Gegen diese Schmerzen hielt er körperliche Anstrengungen für wohltätig und machte deswegen, und natürlich auch aus anderen Gründen, im März und April 1909 eine sehr anstrengende und selbst mit Lebensgefahr verbundene Reise auf einem kleinen Fischkutter nach Island. Diese Reise hat er sehr anschaulich, lebendig und fesselnd im Daheim beschrieben, in welchem Blatte sie nach seinem Tode erschien (Jahrgang 1911, Nr. 29ff.). Die Ernährung auf dieser Reise bestand in einfachster. Matrosenkost, bekam ihm aber gut, wie er auch überhaupt die Reise sehr gut überstand.

1) Die Figuren (Photogramme) verdanke ich der Güte der Herrn Privatdozenten Dr. Sioli, hier. 
Etwa 4 Wochen vor seiner Aufnahme in die medizinische Klinik, die am16. XII. 1909 erfolgte, hatten sich, ohne je de Vorkrankheit, auch ohne Influenza, die damals nicht grassierte, Sprachstörungen aphasischdysarthrischer Art oingestellt, die allmählich an Stärke zunahmen und nicht nach Art eines Schlaganfalles entstanden waren. Der Kranke mußte nach dem Ausdrucke suchen und sprach langsamer als früher. Daneben Schwächeerscheinungen im rechten Arm, Schreibstörungen und Unsicherheit beim Gehen. Niemals Koptweh oder Schwindel oder Erbrechen. Sehen gut:

Lues und Potus negiert. Kein mechanisches Trauma. Hat stark geraucht, besonders seit seinem 30 . Lebenjahre.

Die Aufnahmeuntersuchung am 16. XII. 1909 ergab kräftigen Körperbau und guten Ernährungszustand. Keine nachweisbaren Veränderungen an den inneren Organen. Körpertemperaturen bis zum Anfang Januar 1910 leicht subnormal.

Die Pupillen etwas eng, normal reagierend. Beim Blicken nach außen deutlicher Nystagmus, besonders beim Blick nach rechts. Keine Augenmuskellähmung. Die rechte Sehnervenpapille nach der Untersuchung von Herm Prof. Hummelsheim, ausgesprochen grau" gefärbt, aber ohne Zeichen von Schwund. Die linke zeigt ebenfalls eine deutliche graue Beimengung im Farbenton, aber noch ins Gelbrötliche spielend. Auch an ihr keine Atrophie. Der Papillenrand beiderseits scharf. Sehschärfe rechts $6 / 6$, links $6 / 8$. Gesichtsfeld für Weiß und Farben normal. Keine Skotome.

Deutliche leichte Parese der rechten Extremitäten mit leichter Steigerung einzelner Sehnenreflexe. Kein Großzehenstreckreflex. Deutliche Störung des Lagegefühls der rechten Finger bei Erhaltensein der Schmerzund Berührungsempfindung. Die letztere vielleicht etwas abgeschwächt. Am rechten Fuß und Unterschenkel geringfügige Abschwächung der $\mathrm{B} \varepsilon-$ rïhrungs- und Schmerzempfindung.

Fazialisgebiet und Zunge frei, sie zittert beim Herausstrecken etwas. Starke aphasisch-dysarthrische Sprachstörung; so daß die Verständigung schwierig ist. Kein deutliches Skandieren.

Keine Zeichen von Arteriosklerose und Herzhypertrophie. Der Harn ohne EiweiB und Zucker. Die Wassermannsche Reaktion im Blute regativ.

Im Laufe der nächsten Woche ziemlich rasches Fortschreiten sowohl der rechtsseitigen Parese als der Sprachstörung. Die Sehnenreflexe viel deutlicher gesteigert wie zuerst; an den Armen beiderseits. Rechts jetzt deutlicher Babins zi. Das Lagegefühl der rechten Hand und Finger schwer gestört, aber die übrigen Empfindungsarten wie $t$ ' 'er. Die Sprachstörung hat ebenfalls weiter zugenommen.

Niemals Kopfweh, Schwindel oder Erbrechen.

Anfang Januar 1910 vollständige Lähmung des rechten Armes und seit dem 8. I, auch des rechten Beines mit starken Spasmen. Die Berührungsempfindung an der rechten Hand etwas abgestumpft. Kein Kopfweh.

Die Lumbalpunktion ergibt einen Liquordruck von 110 Atm., 
klare, ganz schwach eiweißhaltige Flüssigkeit, die leider nicht auf Wassermann untersucht wurde.

Die Sprachstörung so stark, daß nur mit größter Mühe eine Verständigung möglich ist. Seelische Niedergeschlagenheit, Klagen über Mattigkeit. Verfallenes Aussehen.

Mitte Januar zeitweilige, an Chorea erinnernde Muskelunruhe, die besonders bei den Bemühungen zu sprechen ausgelöst wird. Der rechte Kornealreflex deutlich herabgesetzt, der linke normal. Im rechten Bein starke, kaum zu überwindende Spasmen, Fußklonus; auch links Steigerung des Patellarreflexes. Bauchdeckenreflexe fehlen völlig. Die rechten Armreflexe gesteigert. Die Lagegefühlsstörung an den Fingern und an der Hand scheint noch zu bestrehen.

Später fehlt der rechte Kornealreflex vollständig; die Zunge weicht etwas nach rechts ab. Das Schlucken etwas erschwert.

Am 20. I. setzt Fieber ein, auf Bronchopneumonien beruhend. Das Schlucken sehr erschwert. Sehr elendes Aussehen; starke Blasenschwäche. Verständigung unmöglich, da auch das Schreiben mit der linken Hand nicht gelingt. Die Psyche erscheint nicht ganz frei.

Am 22. I. vollkommene Schlucklähmung. Das Sprechvermögen aufgehoben; Zunge nicht deutlich bewegbar. Incontinentia urinae et alvi. Temperatursteigerung bisi $: 38 ; 7$. - Am 23. I. völlige Bextußtlosigkeit, Trachealrasseln. Am 24.I. 1910 die Spasmen rechts völlig verschwunden. Temperatur 40,1. Tod nachmittags $2 \mathrm{Uhr}$, nach etwa zehnwöchentlicher, stetig fortschreitender Erkrankung.

Die Diagnose schwankte zwischen enzephalitischen Herden und multipler Sklerose, also nur in ätiologischer nicht in anatomischer Richtung. Tumoren mußten wegen des dauernden Fehlens von Kopfweh, Erbrechen, Stauungspapille und wegen des normalen Druckes der Spinalflüssigkeit ausgezchlossen werden. Lues war nicht nachzuweisen.

Als erstes Zeichen der Erkrankung konnten, vielleicht die gelegentlichen Anfälle von vorübergehender lin ksseitiger Armschwäche gelten, die aber mit seelischen Erregungen zusammenhingen. Die langjährigen Tic- oder Choreaerschemungen haben natürlich mit der zuletzt vorliegenden organisch bedingteg Erkrankung nichts zu tun. Der sichere Anfang des tödlichen Leidens geht erst auf Mitte November 1909 zurück.

Die Sektion (Geh. Rat Ribbert) ergab: Im Sinus longit. sehr viel dunkles, geronnenes Bl t. Die Innenfläche der Dura glatt und feucht. Pia im ganzen nicht getrübt, hinten ödematös; dort einzeln in Gruppen stehende grauweiße Gebilde. An der Schädelbasis gelbe, klare Flüssigkeit. In der vorderen und mittleren Schädelgrube finden sich weiche, sehr wenig festhaftende pachymeningitische Häute von sehr geringer Ausdehnung. In der sonstigen Sinus flüssiges Blut. - Die Hirnsubstanz mit zahlreichen grauen Herden durchsetzt, namentlich in den hinteren Teilen des linken Frontallappens. „Auch das Rückenmark zeigt solche Herde.“ 
Die sonstige Sektion nicht gestattet.

Die Untersuchung von großen gehärteten und gefärbten Schnitten, die

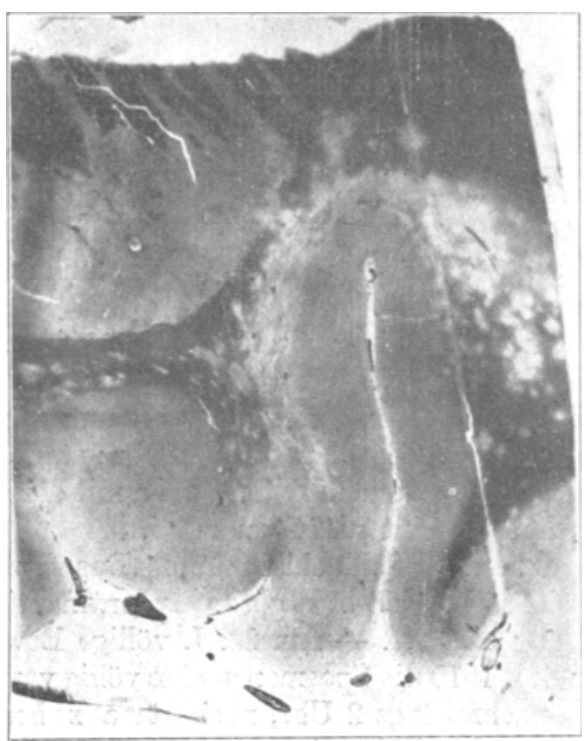

Fig. 1 .

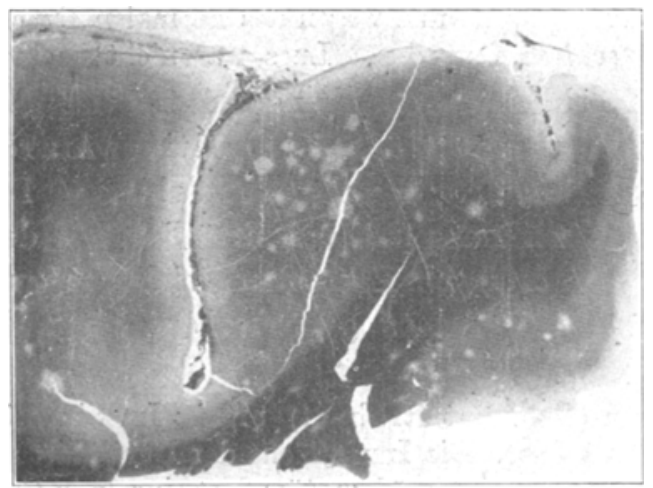

Fig. 2.

ich der Güte des Herrn Prof. Sturs be ró verdanke, ergab eine Unzahl kleiner und kleinster, stecknadelkopf- und. hirsekorngroßer (,miliarer") Herde vor allem in der Marksubstanz der linken Großhirnhälfte, aber auch, wenn auch nicht so häufig, in der Rinde (Fig. 1 i.2). Sie bieten makroskopisch 
das gleiche Bild, wie z. B. das von W. Spielme yer remer Abhandlung über Fnzephalitis beigegebene ${ }^{1}$ ), und findet sich im Kleinhirn nur vereinzelt, anders wie bei Spielmeyer, in dessen Fall sie auch in diesem Gehirnteil häufig vorkommen. Ebenso sieht man sie im Pons nur ganz vereinzelt, und zwar beiderseits. Auch in der Medulla oblong., in der Höhe der Oliven, sind sie nur selten, aber weder in ihrem Pyramidenabschnitt, noch in den Oliven aufzufinden.

Das Rückenmark erwies sich in Abweichung vom makroskopischen

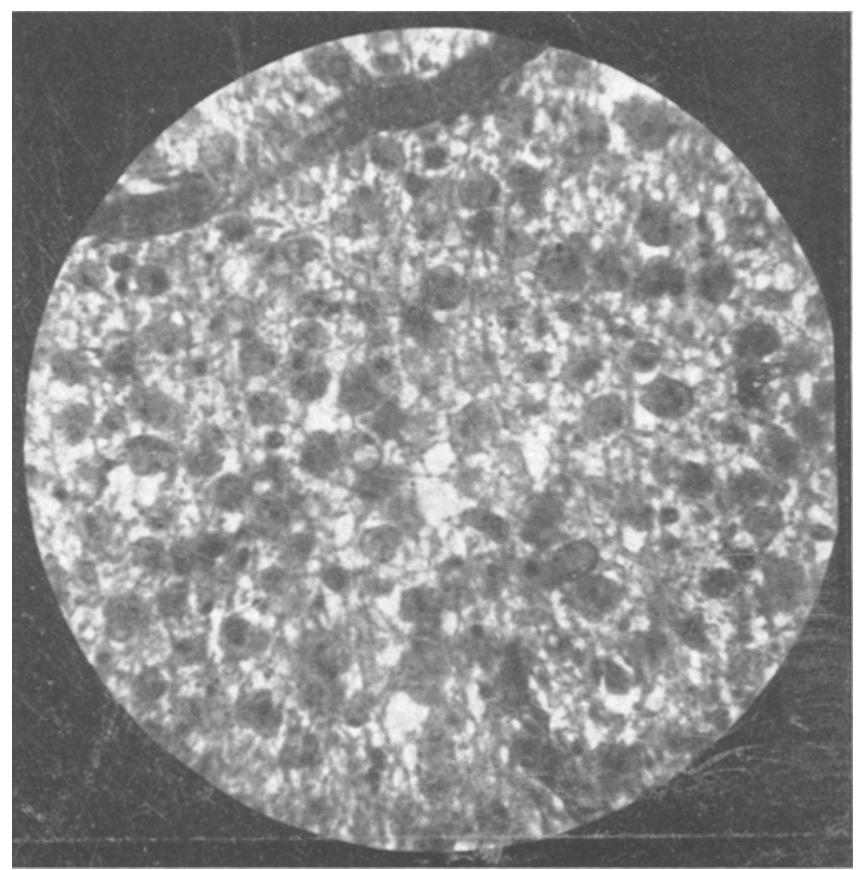

Fig. 3.

Sektionsbefund als völlig normal, sowohl im Hals-, wie im Dorsal-; wie im Lendenteile. Allerdings wurden nur einzelne Querschnitte in den verschiedenen Höhen untersucht. Von einer sekundären Degeneration, besonders auch auf der rechten Seite, ist nichts zu sehen, auch mikroskopisch nicht, trotz der vorhanden gewesenen rech tsseitigen Lähmung.

Mikroskopisch zeigte sich die Pia mater sowohl des Gehirns als des Rückenmarks ganz normal, bis auf starke Erweiterung und erheblicher Blutanfüllung an einzelnen Stellen, und abgesehen von geringen Blutungen, die auch ebenso wie die ganz vereinzelten Blutungen im Gehirne selbst auf

1) W. Spielmeyer, Arch. f. Psych. u. Neurol. Bd. 35, Tafel XV, 1902. 
die Stauungsvorgänge infolge der zuletzt vorhandenen Lungenentzündung bei dem Kranken bezogen werden könnten.

In der Hirnrinde außerhalb der erwähnten kleinen Herde nichts Abnormes nachweisbar, vor allem keine Veränderungen wie bei der progressiven Paralyse.

In den meisten Herden innerhalb der weißen Substanz ist vor allem auffallend eine große Menge dicht aneinandergedrängter Körnchenzellen (s. Fig. "3). Nur in manchen Herden ist die Anzahl dieser Zellen gering;

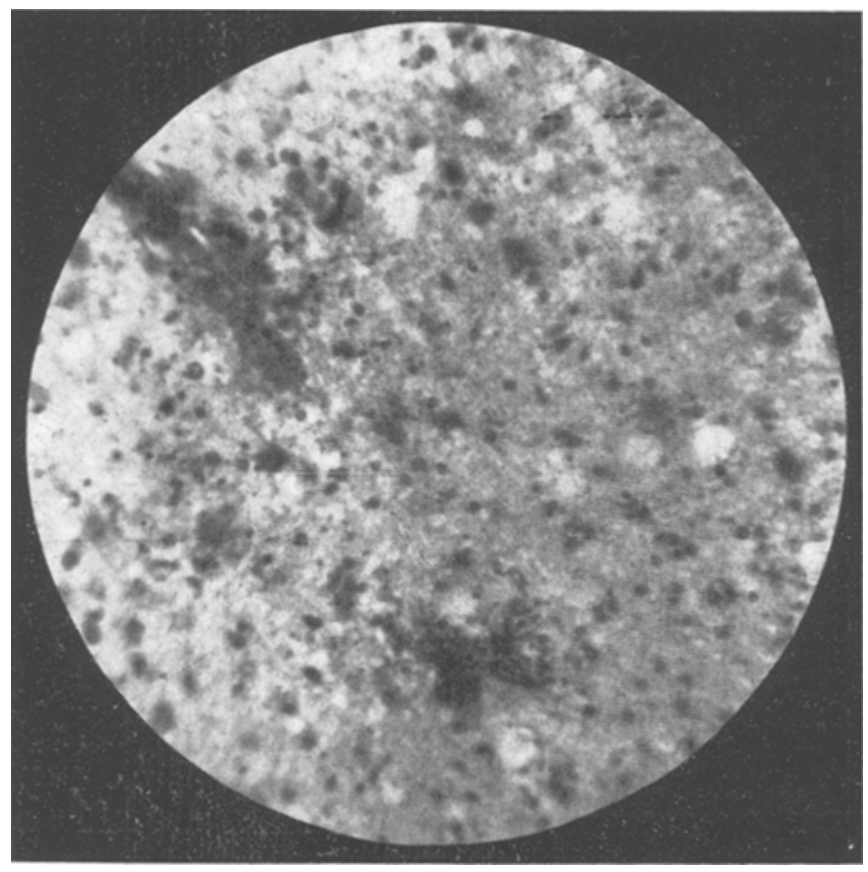

Fig. 4.

auch in den Rindenherden finden sie sich. Die Begrenzung der Herde ist bald scharf, bald mehr verwaschen. In ihnen fehlen fast stets die Markscheiden völlig; in einzelinen kleineren ziehen noch vèreinzelte hindurch.

Herr Privatdozent Dr. Sioli hier, den ich um seine Ansicht bat, fand in den meisten Herden eine mäßige Menge, gemästeter" Gliazellen, in denjenigen mit geringer $m$ Körnchenzellengehalt lebhafte re Gliawucherung besonders am Rande der Herde.

Über das Erhaltenbleiben von Achsenzylindern ist leider an den vorhandenen Präparaten nichts Sicheres auszusagen ${ }^{1}$ ). Nur fand Herr

1) Das Gehirn und Rückenmark geriet während des Krieges in Verlust, so daß weder eraeute Untersuchungen auf Achsenzylinder, noch, wie beabsichtigt, auf etwaige Mikroorganismen mehr angestellt werden konnten. 
Dr. Sioli in einigen kleinen Herden (bei v. Gies son-Färbung) eine Anzahl von Achsenzylindern erhalten. Auch in den Rinde nhe rde n erschien neben dem Ausfall der Markscheiden cin verhältnismäBiges Erhaltenbleiben von Achsenzylindern feststellbar, daneben aber auch ein Ausfall von Ganglienzellen. In einzelnen dieser Herde, ebenso wie in einigen Markherden keine Körnchenzellen, sondern nur Nervenfaserausfall (bloß Markscheidenausfall?) und Gliawucherung. Eine Spirochätenfärbung gelang bei dem Zustande der Präparate nicht mehr.

An den Blutgefäßen konnte ich im allgemeinen ebensowenig wie

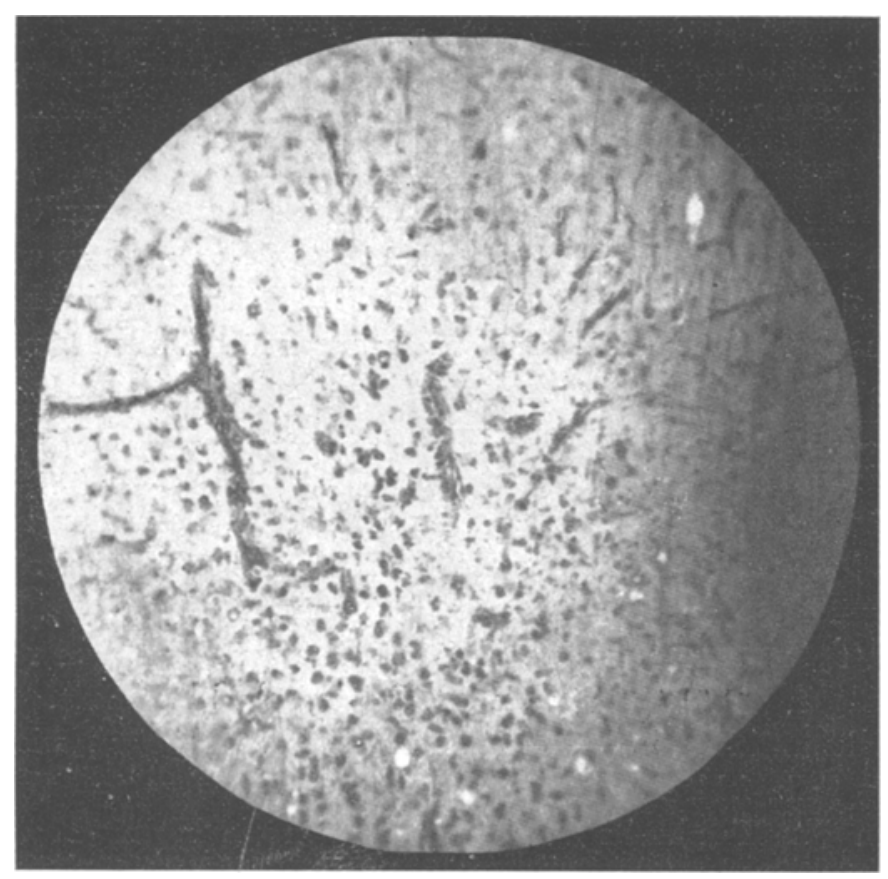

Fig. 5

Herr Dr. Sioli auch in den stark veränderten Herden Veränderungen finden, vor allem keine sogenannte kleinzellige Infiltration, nicht einmal eine besondere Anhäufung mit Körnchenzellen in den perivaskulären Räumen. Die Gefäßscheiden sind zum kleinen Teile mit roten Blutkörpern angefüllt. Nur an ganz vereinzelten Stellen eine leichte Infiltration mit Rundzellen und nach der Meinung des Herrn Dr. Sioli auch eine Endothelkernanschwellung.

Anatomisch ist also, vor allem wegen der auBerordentlich geringen Beteiligung. der BlutgefäBe an dem Krankheitsprozeß, 
nicht das schematische Vollbild einer akuten oder subakuten Entzündung, einer Enzephalitis gegeben, sondern dasjenige einer herdförmigen Gewebszerstörung. Faßt man die Körnchenzellen als veränderte Leukozyten auf, so muß man wenigstens von einer reaktiven Entzündung sprechen. Jedenfalls lag keine eitrige Enzephalitis vor. An die sogenannte akute hämorrhagische Enzephalitis erinnert sebr die ,Flohstich"form der Herde und das vorzugsweise Befallensein des Großhirns. Aber is fehlen die vielfachen Blutungen, die nur in sehr geringem Maße vorhanden sind. Alle Herde erschienen nicht rot, sondern grau. Immerhin war wenigstens hier und da eine geringe Infiltration der Gefäße mit Rundzellen und eine leichte Blutung vorhanden, so daß man die Erkrankung als leichteste Form dieser Enzephalitis bezeichnen könnte, falls man nicht die leichto Gefäßveränderung und die geringe Blutung als etwas Sekundäres betrachten will oder muß. Immerhin könnte man die Annahme machen, da $B$ die gleiche Schädlichkeit, die in so vielen Fällen von kleinherdiger hämorrhagischer Enzephalitis zu den Blutungen führt, in anderen Fällen nicht so konzentriert oder nicht in solcher Menge einwirkt, daß sie eine blutige Entzündung macht, sondern tben nur eine viel leichtere einfache. Der klinische Befund spricht womöglich noch mehr gegen eine hämorrhagische Enzephalitis als der anatomische, ebenso auch gegen die etwaige Annahme, daß eine Heine-Medinsche Gehirnentzündung vorgelegen habe. Denn es fehlte vor allem der akute Beginn des Leidens mit sofort einsetzenden schweren Krankheitssymptomen, ferner Kopfweh und ein erhöhter Druck der Zerebrospinalfliussigkeit. Fieber kann bekanntlich bei beiden Erkrankungsformen fehlen, wie es in unserem Falle fehlte. Jedentalls spricht aber das stetige Vorwärtsschreiten der Krankheitserscheinungen gegen die Heine-Medinsche Krankheit.

Eine Embolisierung und, Thrombosierung kann nicht angenommen werden, da für sie jeder Anhaltspunkt fehlt und der anatomische Befund gegen sie spricht.

So wird man auf die Annahme einer örtlichen direkten sehweren, fortschreitenden Schädigung der Gehirnsubstanz hinge wiesen, wie anch mein verehrter Herr Kolle ge Ribbert annimmt, dem inh die Präparate zeigte. Man wird durchaus an die erwähnten eigentümlichen Herde erinnert, dje Spielmeyer bei der progressiven Paralyse gefunden hat; die doch unzweifelhaft durch die Einwirkung der Spirochaeta pallida und ihrer Gifte hervorgerufen werden. 
Es kann aber in unserem Falle nach der Krankengeschichte und auch nach dem anatomischen Befunde an den Gefäßen und an den Meningen eine Lues nicht angenommen werden. Es bleibt deswegen fast nur übrig, an eine akute oder subakute multiple Sklerose zu denken, falls man nicht eine bisher noch ganz unbekannte Kategorie von Erkrankungen annehmen will.

Die ziemlich langsame Entwicklung des Leidens und ihr weiteres Fortschreiten bis zum Tode auch innerhalb von Monaten ist bei dieser Krankheit bekannt, ebenso wie der bei ihr häufig vorhandene Mangel an Fieber. Auch der anatomische Befund entspricht makroskopisch ganz dem Verhalten bei der multiplen Sklerose, mit Einschluß des Mangels von sekundärer Degeneration.

Der Untergang verhältnismäßig vieler Achsenzylinder in unserem Falle kann um so weniger entscheidend sein, als, wie erwähnt, Siemerling und Raecke behaupten, daß bei der multiplen Slklerose gerade die erste mikroskopisch kleine Herdbildung mit umschriebenem Zerfall von Achsenzylindern beginnt, dem sich erst ein umfassenderer Untergang von Markscheiden anschließt. Im übrigen gleicht das Verhalten der Herde in unserem Falle mit seiner häufig so scharfen Abgrenzung von dem normalen Gewebe und mit seinen Körnchenzellen durchaus dem Befunde wie bei multipler Sklerose in seinen früheren Stadien. Die Blutgefäße haben sich auch nicht selten bei der multiplen Sklerose nicht mitbeteiligt gefunden. Nebenbei gesagt: Wenn man vielfach liest, daß daraus, daß sich auf Querschnitten mitten im sklerotischen Herd ein verdicktes Gefäß vorfindet, folge, daß diese GefäBveränderung die benachbarte Gewebsveränderung hervorgebracht habe, so wird dabei vergessen, daß diese Gefäßveränderung nicht gut diese Nachbardegeneration hervorrufen kann, sondern eventuell eine Entartung viel weiter abwärts oder aufwärts gelegener Bezirke, die von diesem Gefäß versorgt werden.

Es ist also durchaus nicht unwahrscheinlich, daß es sich in unserem Falle um eine Schädigung des Großhirns durch den Erreger der multiplen Sklerose handelte, der allerdings zurzeit noch nicht sicher und in unserem Falle überhaupt nicht nachgewiesen ist.

In dem erwähnten Spielmeyerschen Falle war zunächst klinisch eine gewisse Ähnlichkeit mit dem unsrigen vorhanden: In negativer Richtung das Fehlen einer nachweisbaren Ursache bei einem allerdings bereits 59 jährigen Manne, fortschreitender Verlauf während einiger Monate mit Krampfanfällen, ohne Kopfweh, und zuletzt rascheres Vor- 
wärtsschreiten der Erkrankung mit ganz ähnlichen schwersten Sprechstörungen wie in unserem Falle, nebst leichter Lähmung im rechten Fazialis und Hypoglosus. Anatomisch aber außer den erwähnten ganz gleich gestalteten miliaren Herden im Großhirn nud Kleinhirn stärkere ,entzündliche" Veränderungen, wie ausgedehntere Gefäßinfiltrationen, stärkere Gliawucherung, andererseits eine viel geringere Körnchenzellenanhäufung, also schließlich nur graduelle Unterschiede.

In einem Falle von Schlagenhaufer ${ }^{1}$ ), bei dem allerdings nur das Rückenmark untersucht wurde und den er als einen Fall von ,subakuter inselförmiger Sklerose" beschreibt und dcr nach $7 \frac{1}{2}$ Wochen zum Tode führte, wurden in den aufgefundenen Herden , unstreitige Zaichen einer Entzündung vermißt", a ber es bestand ein beträchtlicher Zerfall von Markscheiden mit Erhaltenbleiben zahlreicher nackter Achsenzylinder und fehlender sekundärer Degeneration, sowie eine Gliawucherung. Aber es war eine, starke kleinzellige Infiltration der Adventitialpartien der Gefäße" vorhanden. Im ganzen also ebenfalls eine nicht unbedeutende Ähnlichkeit mit unserem Falle.

\section{Nachtrag.}

Von der neuerdings von Economo ${ }^{2}$ ), S. A. K. Wilson ${ }^{3}$ ) und anderen, zuletzt von Reinhart4) beschriebenen ,Encephalitis lethargica" oder "Epidemic encephalitis" (Wilson) unterscheidet sich unser Fall klinisch und nach den bisber bekannt gewordenen Befunden auch anatomisch ganz wesentlich. Abgesehen von der nicht entscheidenden Tatsache; daß unser Fall ganz sporadisch auftrat und sicher mit epidemischer Grippe nichts zu tun hatte, die damals nicht herrschte, war kein akuter Beginn mit Kopfweh und Übelkeit vorhanden. Es fehlte aber vor allem jene eigentümliche Verbindung von Schlafsucht und Augenmuskellähmung, wie bei den von den genannten Klinikern beschriebenen Fällen. Anatomisch waren in zweien der von Reinhart aus der Kieler medizinischen Klinik mitgeteilten Fällen nach einer vorläufigen. Mitteilung zwar umschriebene Herde vorhanden, in Form von "flohstichartigen". Blutungen und vereinzelten „,kleinen Nekrosen mit reaktiver Gliawucherung", sowie von. kleinen

1) Arbeiten aus dem Neurol. Institute in Wien 1900, S. 223.

2) Economo, Wiener klin. Wochensher. 1917, Nr. 30, S. 581.

3) Reinhart, Deutsche med. Wochenschr. 1917, Nr. 19, S. 514.

4) S. A. K. Wilson, Epidemic encephalitis. Lancet 1918. Juni. 
Entzündungsherden. Der Hauptort dieser Veränderungen befand sich aber ganz anders wie in unserem Falle im zentralen Höhlengrau des 3. und 4. Ventrikels, sowie des Aquaeductus Sylvii. Es war also eine Polioenzephalitis vorhanden, die aber nach der Meinung von James ${ }^{1}$ ) nichts mit der gewöhnlichen epidemischen Poliomyelitis zu tun hat, während sie Economo in Analogie mit der Heine-Medinschen Krankheit bringt. Die etwaigen Beziehungen dieser letztgenannten Erkrankung zu meinem Falle sind bereits oben erörtert worden.

Sowohl von Jores als von Wilson wurden perivaskuläre Infiltrationen, von Wilson auch mehr oder weniger schwere meningeale Veränderungen neben Zellinfiltrationen in der Hirnrinde gefunden, alles Zustände, die in unserem Falle fehlten, vielleicht weil nicht mehr die Anfänge der Erkrankung vorlagen, die aber andererseits noch bis zum Tode stetig neue Fortschritte gemacht hatte, also sehr wohl auch neue Entzündungsherde erzeugen konnte.

I) J. P. James, Lethargic encephalitis. Lancet 1918. Letztere Abhandlungen nach Referaten zitiert.

\section{Nachtrag.}

Herr Dr. Sioli konnte auch bei erneuter Untersuchung mit Spirochätenfärbung und Bielschowsky-Färbung keinen andern Befund als trüher erheben. Die Zahl der Achsenzylinder in den Herden ist nur in geringem Maße verringert.

Figurentafel (Photogramme).

Fig. 1 u. 2. Schnitte aus den Großhirnwindungen mit vielen kleinen, in Fig. 1 zum Teile zusammengeflossenen Herden.

Fig. 3. Vergr. $170 \mathrm{mal}$, van Gieson-Färbung: Körnchenzellenanhäufung mit „gemästeten“ Gliazellen.

Fig. 4. Vergrößerung 170 mal, van Gieson-Färbung: Kleine Blutung, geringe Gliawucherung; keine Körnchenzellen.

Fig. 5. Vergrößerung 50 mal, Marchi-Präparat. Kleiner Herd in der Hirnrinde mit Körnchenzellen (durch Marchi schwarz gefärbt). Noch erhaltene Markfasern und Ganglienzellen (schwach schattenartig gefärbt). 